\title{
Block Copolymer Micelles as Switchable Templates for Nanofabrication
}

\author{
Sivashankar Krishnamoorthy, ${ }^{\dagger}$ Raphaël Pugin, ${ }^{\dagger}$ Juergen Brugger, ${ }^{\ddagger}$ Harry Heinzelmann, ${ }^{\dagger}$ \\ Arno C. Hoogerwerf,,$^{\dagger}$ and Christian Hinderling, ${ }^{*}$ \\ Centre Suisse d'Electronique et de Microtechnique SA, Jaquet Droz 1, CH-2007 Neuchâtel, Switzerland, \\ and Ecole Polytechnique Fédérale de Lausanne (EPFL), Microsystems Laboratory (LMIS), \\ CH-1015 Lausanne, Switzerland
}

Received August 23, 2005. In Final Form: February 17, 2006

\begin{abstract}
Block copolymer inverse micelles from polystyrene-block-poly-2-vinylpyridine (PS- $b$-P2VP) deposited as monolayer films onto surfaces show responsive behavior and are reversibly switchable between two states of different topography and surface chemistry. The as-coated films are in the form of arrays of nanoscale bumps, which can be transformed into arrays of nanoscale holes by switching through exposure to methanol. The use of these micellar films to act as switchable etch masks for the structuring of the underlying material to form either pillars or holes depending on the switching state is demonstrated.
\end{abstract}

Block copolymers have been explored extensively as templates for nanofabrication. ${ }^{1-3}$ Nanostructures form as a result of the microphase separation driven by chemical incompatibility between the constituent blocks of the copolymer. The morphology and dimensions of the nanostructures thus obtained are tunable with the suitable choice and length of the constituent polymer blocks. Nanoscale polymer etch masks were obtained from microphase-separated block copolymer thin films by selective degradation and removal of one of the blocks, whereas the other block serves as a lithographic mask to etch the underlying substrate. ${ }^{4-7}$ Surface micelles that form through the preferred adsorption of one polymer block onto the surface have also been used to structure surfaces, and the as-coated film offers an inherent mass thickness contrast necessary for transferring structures by etching. ${ }^{8,9}$ Block copolymers are furthermore well known to form micelles in solution when dissolved in selective solvents. ${ }^{10,11}$ The soluble block forms a swollen corona in the solution phase shielding the insoluble block that forms a highly condensed core. These micelles can be spherical, cylindrical, or wormlike depending on the block ratios, the interfacial energy between the blocks, and the solvent quality. There have been several reports on the deposition of spherical micelles on surfaces to obtain arrays of functional centers that were used for making nanoparticle arrays $^{12-14}$ and other functional ${ }^{15}$ or responsive ${ }^{14,16}$ surfaces.

* Corresponding author. E-mail: christian.hinderling@csem.ch.

$\succ$ Centre Suisse d'Electronique et de Microtechnique SA.

†cole Polytechnique Fédérale de Lausanne (EPFL).

(1) Hamley, I. W. Nanotechnology 2003, 14, R39-R54.

(2) Park, C.; Yoon, J.; Thomas, E. L. Polymer 2003, 44, 6725-6760.

(3) Forster, S.; Antonietti, M. Adv. Mater. 1998, 10, 195-217.

(4) Cheng, J. Y.; Ross, C. A.; Chan, V. Z. H.; Thomas, E. L.; Lammertink, R. G. H.; Vancso, G. J. Adv. Mater. 2001, 13, 1174-1178.

(5) Guarini, K. W.; Black, C. T.; Zhang, Y.; Kim, H.; Sikorski, E. M.; Babich,

I. V. J. Vac. Sci. Technol. 2002, 20, 2788-2792.

(6) Lammertink, R. G. H.; Hempenius, M. A.; van den Enk, J. E.; Chan, V.

Z. H.; Thomas, E. L., and Vancso, G. J. Adv. Mater. 2000, 12, 98-103.

(7) Park, M.; Harrison, C.; Chaikin, P. M.; Register, R. A.; Adamson, D. H. Science 1997, 276, 1401-1404.

(8) Spatz, J. P.; Eibeck, P.; Mossmer, S.; Moller, M.; Herzog, T.; Ziemann, P. Adv. Mater. 1998, 10, 849-852.

(9) Meli, M. V.; Badia, A.; Grutter, P.; Lennox, R. B. Nano Lett. 2002, 2, $131-135$.

(10) Riess, G. Prog. Polym. Sci. 2003, 28, 1107-1170.

(11) Moffitt, M.; Khougaz, K.; Eisenberg, A. Acc. Chem. Res. 1996, 29, 95102.

(12) Kastle, G.; Boyen, H. G.; Weigl, F.; Lengl, G.; Herzog, T.; Ziemann, P.; Riethmuller, S.; Mayer, O.; Hartmann, C.; Spatz, J. P.; Moller, M.; Ozawa, M.; Banhart, F.; Garnier, M. G.; Oelhafen, P. Adv. Funct. Mater. 2003, 13, 853-861.
Micellar films loaded with metal salts or nanoparticles derived from them have been used as etch masks to structure the underlying substrate. In the remarkable case of PS- $b-\mathrm{P} 2 \mathrm{VP}$ micelles loaded with $\mathrm{AuCl}_{4}{ }^{-}$, the authors found an inversion of the relative etch rates upon reducing the included salt to gold nanoparticles, allowing the inversion of the etch contrast depending on the redox state of the mask. ${ }^{17}$ We report in this letter the responsive behavior of an amphiphilic diblock copolymer micellar array that can be switched between two complementary surface topographies and polarities by simple means as well as its use as an etch mask for the structuring of the underlying silicon substrate. The complementary topographies obtained from the micellar film lead to complementary topographies in the silicon, resulting in either arrays of pillars or holes depending on the switching state of the micellar film (Figures 1, 3, and 4). We emphasize that the use of films of block copolymer micelles and thin films of microphase-separated block copolymers for surface structuring is not the same and experimentally the former offers several attractive advantages. Micelles offer the flexibility to be deposited on a variety of surfaces and over large areas relatively easily. In addition, the micelle preparation and coating conditions can be varied to tune the nanostructure dimensions and the lateral 2D periodicities, without having to change the polymer molecular weight or block ratios. ${ }^{18}$ We highlight that the novelty in our approach is the use of switchable topographies obtained by exploiting the responsiveness of the micellar films to achieve complementary structuring in the nanoscale, with added process advantages such as easy scalability and fabrication.

Monolayers of poly(styrene-block-2-vinylpyridine) (PS- $b$ P2VP) (91 500- $b$-105 000 g/mol, PDI 1.1) micelles were prepared by spin casting a toluene $0.5 \mathrm{wt} \%$ solution of the diblock copolymer at $2000 \mathrm{rpm}$ on freshly piranha-treated silicon surfaces. In toluene, a solvent for PS and a nonsolvent for P2VP, micelles with a PS corona and P2VP core form. A radius of gyration $\left(R_{\mathrm{g}}\right)$

(13) Spatz, J. P.; Mossmer, S.; Hartmann, C.; Moller, M.; Herzog, T.; Krieger, M.; Boyen, H. G.; Ziemann, P.; Kabius, B. Langmuir 2000, 16, 407-415.

(14) Boontongkong, Y.; Cohen, R. E. Macromolecules 2002, 35, 3647-3652.

(15) Meiners, J. C.; Elbs, H.; Ritzi, A.; Mlynek, J.; Krausch, G. J. Appl. Phys. 1996, 80, 2224-2227.

(16) Webber, G. B.; Wanless, E. J.; Armes, S. P.; Biggs, S. Faraday Discuss. 2004, 128, 193-209.

(17) Spatz, J. P.; Herzog, T.; Mossmer, S.; Ziemann, P.; Moller, M. Adv. Mater. 1999, 11, 149-153.

(18) Krishnamoorthy, S.; Pugin, R.; Heinzelmann, H.; Brugger, J.; Hinderling, C. Adv. Funct. Mater. 2005, accepted for publication. 


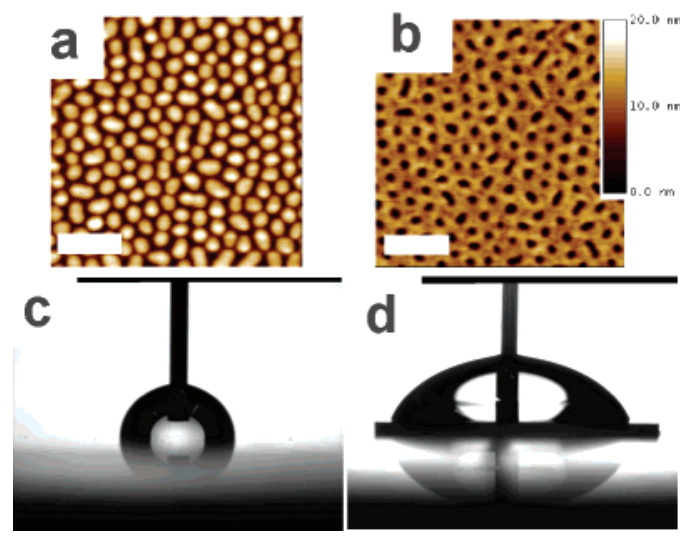

Figure 1. (a) AFM micrograph of the PS- $b$-P2VP micellar film as coated and (b) the micelle-coated substrate treated with methanol for $30 \mathrm{~s}$. The scale bars are $250 \mathrm{~nm}$ each. (Bottom) optical images of advancing water contact angles (c) on as-coated $\left(\theta_{\text {adv }} 90 \% \theta_{\text {rec }}\right.$ $\left.65^{\circ}\right)$ micelles and (d) on micelles transformed after methanol treatment $\left(\theta_{\text {adv }} 65^{\circ} / \theta_{\text {rec }}<20^{\circ}\right)$.

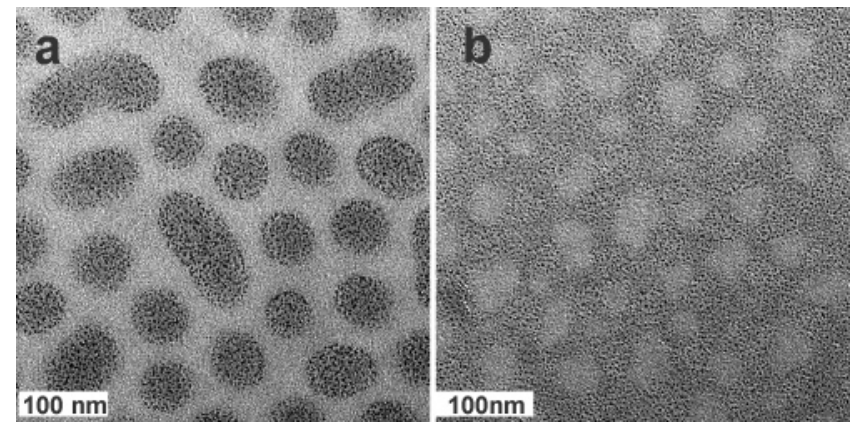

Figure 2. TEM micrographs of (a) as-coated PS- $b$-P2VP micelles and (b) the transformed micellar film after methanol treatment on a carbon grid, both stained with $\mathrm{HAuCl}_{4}$.

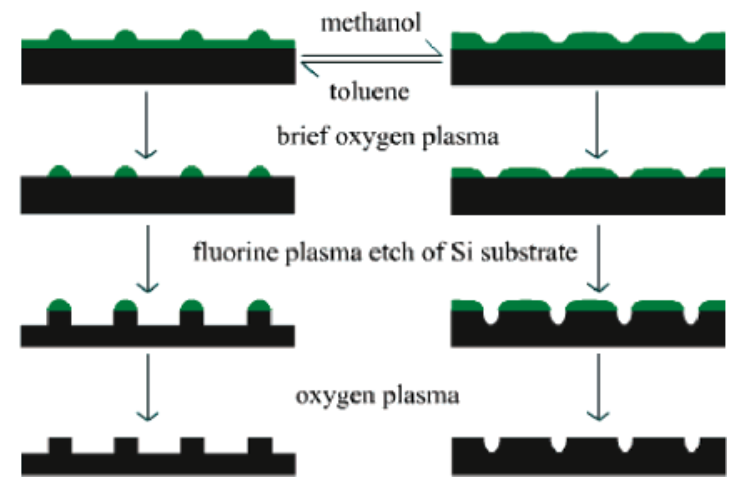

Figure 3. Steps used to create (left) pillars and (right) holes in silicon using polymer masks derived from micelles.

of $57 \mathrm{~nm}$ and a hydrodynamic radius $\left(R_{\mathrm{h}}\right)$ of $68 \mathrm{~nm}$ were determined using static (SLS) and dynamic light scattering (DLS) measurements, respectively. The ratio of $R_{\mathrm{g}}$ to $R_{\mathrm{h}}$ results in a $\rho$ parameter of 0.84 . The slightly higher $\rho$ value of the micelles in comparison to the ideal sphere value of 0.778 indicates a slight deviation from spherical geometry, which is also apparent in the AFM and TEM images (Figures 1a and $2 \mathrm{a}$ ). An $A_{2}$ value of $1.2 \times 10^{-8} \mathrm{~mol} \cdot \mathrm{mL} / \mathrm{g}^{2}$ obtained from the SLS measurements indicates good solvation of the micelles in toluene.

Tapping mode AFM measurements on as-coated substrates show an 11-nm-thick continuous layer, which we attribute to the collapsed coronas of the micelles, from which individual micelles protrude as bumps of $11 \mathrm{~nm}$ height with a periodicity of $72 \mathrm{~nm}$. An apparent micelle diameter (not corrected for tip convolution)
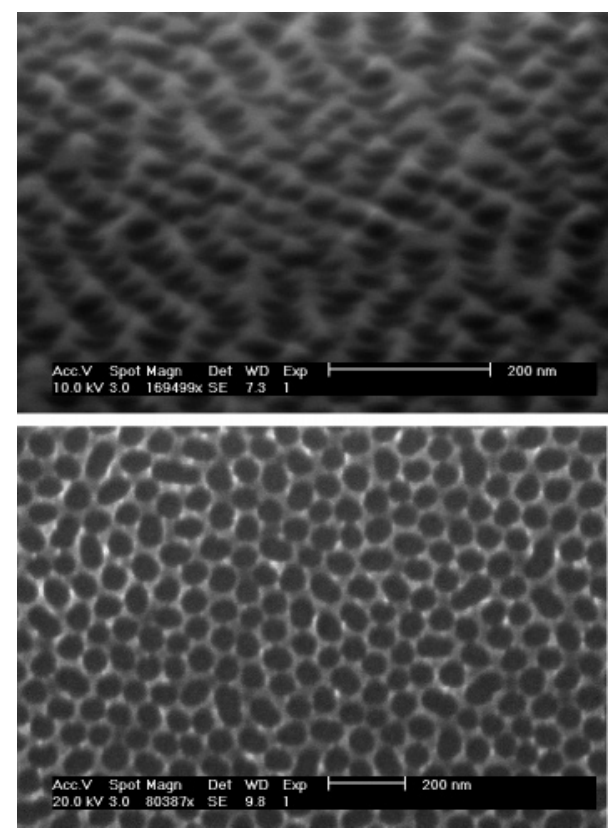

Figure 4. Scanning electron micrographs of (a) pillars and (b) holes etched into silicon.

of $65 \mathrm{~nm}$ was measured with the AFM (Figure 1a). The thickness of the continuous layer was determined by imaging a scratch made with Teflon tweezers exposing the bare silicon surface.

The micellar film undergoes a transformation when dipped into methanol, resulting in an array of holes with an average depth of $14 \mathrm{~nm}$ and the same periodicity as the as-coated micellar array. We find a literature precedence of such micellar thin film transformations upon exposure to solvents. In one of these cases, transformation of a freely floating PS- $b$-P4VP micellar film is effected by liquid-phase exposure to methanol. ${ }^{19}$ In this case, the authors have shown a core-corona inversion to take place upon methanol treatment, which could be reversed by exposure to toluene. In another case, the authors have used methanol vapors to swell a PS- $b$-P2VP micellar film adsorbed on a mica surface. ${ }^{20}$ Their study finds that there is a reversible morphology change upon exposure to methanol or toluene vapor but that the lateral arrangement of the chains is not affected by the vapor treatment and there is no complex rearrangement of the copolymer chains. In our case, we have used a PS- $b$-P2VP micellar film on a silicon surface and find morphology changes similar to those reported by these authors upon dipping the micelle-coated substrate in methanol. In addition, we find that the transformed films obtained upon exposure to methanol yield a surface that is hydrophilic with a contact angle of $65^{\circ}(\mathrm{adv}) /<20^{\circ}(\mathrm{rec})$, in comparison to that of the as-coated micellar film, which gives a contact angle of $89^{\circ}(\mathrm{adv}) / 65^{\circ}(\mathrm{rec})$. (Figure 1$)$. For comparison, we find the respective contact angles on freshly spin-coated films of polystyrene and poly-2-vinylpyridine homopolymers to be $95^{\circ}$ $(\mathrm{adv}) / 65^{\circ}(\mathrm{rec})$ and $64^{\circ}(\mathrm{adv}) /<20^{\circ}(\mathrm{rec})$. The increased hydrophilicity after methanol treatment cannot be explained unless the core-forming P2VP is at least partially exposed to the surface. To better understand the location of the P2VP block in the ascoated and the transformed films, both films were prepared on a carbon-coated copper grid and were stained with $\mathrm{HAuCl}_{4}$ from aqueous solution. $\mathrm{HAuCl}_{4}$ binds selectively to the P2VP block and offers a dark contrast to PVP-occupied areas. A subsequent

(19) Sohn, B. H.; Yoo, S. I.; Seo, B. W.; Yun, S. H.; Park, S. M. J. Am. Chem. Soc. 2001, 123, 12734-12735.

(20) Elbs, H.; Drummer, C.; Abetz, V.; Krausch, G. Macromolecules 2002, $35,5570-5577$. 
TEM investigation showed that the gold particles formed by electron beam reduction are distributed at the location of the micellar cores in the as-coated films and throughout the matrix and not in the holes (Figure 2) in the transformed film. This is indicative of a more complex rearrangement such as a corecorona inversion rather than a simpler transformation as described in ref 20. The topographic reconstruction of the micellar thin films was found to be independent of the substrate and was reproducible on $\mathrm{Si}_{3} \mathrm{~N}_{4}, \mathrm{SiO}_{2}$, quartz glass, DLC, and Au surfaces as well.

The attractive aspect of the experiment is the switchability of the micellar film between complementary topographies and surface polarities, with switching times on the order of a few seconds. The contact angle changes observed were found to be stable and reversible over several cycles of methanol-toluene treatments. This responsive behavior is similar to that exhibited by surfaces modified with grafted amphiphilic block copolymer molecules upon exposure to solvents or temperature. ${ }^{21-28}$ Micelles offer additional advantages for surface modification because they are individual nanoscale entities that can be readily coated over a wide range of substrates.

To transfer the polymeric structures into the underlying substrate, the as-coated and the transformed films were first exposed to a brief oxygen plasma $(30 \mathrm{~W}, 50 \mathrm{mT}, 30 \mathrm{~s})$ to remove the continuous polymer layer between the protrusions and at the bottom of the holes, respectively. This resulted in polymer masks consisting either of 10-nm-high polymer dots with the bare silicon surface exposed between them or of a 10-nm-thick porous polymer mask with pores reaching the silicon surface.

The silicon substrates with the polymer masks were further subjected to a brief $\mathrm{SF}_{6}$ plasma (15 s) to etch the silicon from the exposed regions and to create either pillars or holes in the silicon depending on the initial switching state of the micellar layer (Figure 3). This was followed by an oxygen plasma step to remove any remaining polymer.

The structure of the polymer masks could be transferred into silicon to achieve pillars with a mean height of $26 \mathrm{~nm}$ and holes

(21) Zhao, B.; Brittain, W. J. Prog. Polym. Sci. 2000, 25, 677-710.

(22) Boyes, S. G.; Brittain, W. J.; Weng, X.; Cheng, S. Z. D. Macromolecules 2002, 35, 4960-4967.

(23) Webber, G. B.; Wanless, E. J.; Armes, S. P.; Tang, Y. Q.; Li, Y. T.; Biggs, S. Adv. Mater. 2004, 16, 1794-1798.

(24) Mori, H.; Hirao, A.; Nakahama, S.; Senshu, K. Macromolecules 1994, 27, 4093-4100.

(25) Senshu, K.; Yamashita, S.; Mori, H.; Ito, M.; Hirao, A.; Nakahama, S. Langmuir 1999, 15, 1754-1762.

(26) Russell, T. P. Science 2002, 297, 964-967.

(27) Aoshima, S. and Hashimoto, K. J. Polym. Sci., Part A: Polym. Chem. 2001, 39, 746-750.

(28) Sugihara, S.; Hashimoto, K.; Okabe, S.; Shibayama, M.; Kanaoka, S.; Aoshima, S. Macromolecules 2004, 37, 336-343. with a depth of $27 \mathrm{~nm}$ (Figure 4). We observed a positive tapering profile for the pillars and cup-shaped geometry for the holes from the images recorded with sample tilt and SEM cross-section analysis, respectively. Efforts are underway to increase the etch anisotropy and aspect ratio of the structures by optimizing plasma conditions. Because etch rates of polymers (photoresists) under the conditions employed are at least 10 times smaller than the etch rate of silicon, we deem structures depths of over $100 \mathrm{~nm}$ possible. This would pave the way for numerous applications, for example, cheap nanosieves that have been achieved only by FIB or e-beam processing.

The structure transfer into the underlying silicon substrate was achievable even without going through the short oxygen plasma step described earlier. However, the masks developed this way also offer other attractive possibilities for nanofabrication, such as selective functionalization of the substrate in the exposed regions or site-selective crystallization. ${ }^{29}$

The morphology changes described above were also observed with the micelles prepared with two other molecular weights (190 000- $b-190000$ and $185000-b-32000)$ of PS- $b$-P2VP, offering possibilities for tuning the dimensions and periodicities of the pillar and hole arrays. The contact angle difference between the surfaces could likely be further increased by using a combination of the surface functionalization with an increase in surface roughness by microstructuring.

We have demonstrated in this report the simple switching of monolayers of PS-P2VP micelles between complementary morphologies and different surface energies and their use as masks for nanostructuring silicon surfaces. This led to etch masks that can be switched to result in either pillars or holes in the underlying substrate in a simple manner, which is equivalent to using a hypothetical resist that can be switched between a positive and negative tone. Further work will aim at improving etch selectivity and increasing aspect ratios, using this type of templating for other steps in nanofabrication, and exploring the combination with top-down methods.

Acknowledgment. This work was supported by the National Center of Competence in Research (NCCR) in nanoscale science of the Swiss National Science Foundation for Scientific Research. We thank them for their support. We thank Olivier Casse and Corinne Vebert, University of Basel for their kind help with the light scattering experiments. We thank Pierre-André Clerc of COMLAB, the shared cleanroom of CSEM, and the Institute of Microtechnology of the University of Neuchâtel for assistance with etching.

LA052299A

(29) Barton, J. E.; Odom, T. W. Nano Lett. 2004, 4, 1525-1528. 